\title{
DROWSY DRIVER MONITOR AND WARNING SYSTEM
}

\author{
Richard Grace \\ Robotics Institute \\ Carnegie Mellon University \\ Pittsburgh, Pennsylvania \\ E-mail: rgrace@rec.ri.cmu.edu
}

Summary: The design and use of a low-cost drowsy driver monitor, the Copilot, and proposed driver interfaces are presented. The Copilot consists of a digital camera integrated with a low-cost digital signal processor (DSP). The Copilot is a functionally enhanced version of a previous monitor that has been successfully used in a variety of research projects in simulators and in over-the-road vehicles. Compared to its predecessor, the new monitor is small and easy to use, providing an effective research tool for the field or in the laboratory.

The Copilot measures slow eyelid closures as represented by PERCLOS (Percent Eyelid Closure). PERCLOS is defined as the proportion of time that a subject's eyes are closed over a specified period. PERCLOS has been separately validated in two independent laboratories as an accurate predictor of performance degradation in sleepdeprived subjects.

The current driver interface is based on recent experimental results that drowsiness feedback can reduce drowsiness and improve driver performance for sleep deprived truck

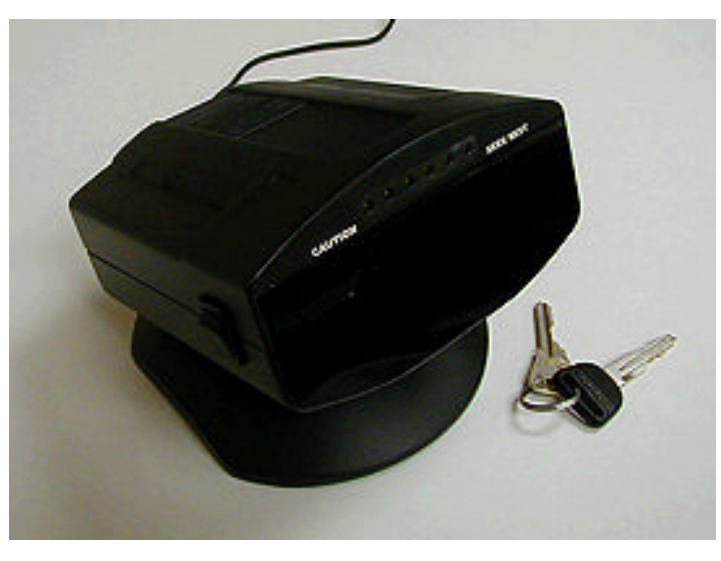
drivers operating a truck simulator. This controlled experiment was undertaken with $\mathrm{n}=16$ Commercial Driving License (CDL) holders driving a high-fidelity truck simulator (TruckSim $\square$ ) to establish the effects of drowsiness feedback on: (1) driver alertness-drowsiness; (2) driving performance and (3) driver-initiated behaviors. Subjects served as their own controls, driving one simulated 4-hr night drive without drowsiness feedback (control condition) and one simulated 4-hr night drive with drowsiness feedback (feedback condition).

Although there was significant between-subject variability in drowsiness and consequently in the number of drowsiness-based alarms and warning alerts, drowsiness feedback tended to have consistent effects on key classes of outcome variables, including reduced drowsiness levels, improved driver performance and self alerting activities (driver movements). The warning triggers are associated with PERCLOS calculated over three minutes. The current interface consists of an audible tone that is associated with the readings of a visual gauge. Work is continuing over the next year to refine the driver interface. 


\section{INTRODUCTION}

Driver drowsiness poses a major threat to roadway safety and the problem is particularly severe for commercial motor vehicle (CMV) drivers. There are approximately 1.6 million truck tractors and 3.6 million trailers used in the motor carrier industry today. CUT's are involved in approximately 200,000 crashes each year. A recent analysis [FHWA, 1998] of the problem size estimates that fatigue related crashes constitute:

- $0.71 \%$ to $2.7 \%$ of all police reported crashes involving CUT's

- $3.2 \%$ to $7.6 \%$ of all fatalities associated with CUT crashes

- $15 \%$ to $36 \%$ of all crashes fatal to the CUT driver

As evident in these statistics, fatigue related crashes tend to be severe. A typical fatigue related crash involves the CUT drifting off the roadway without brake application (run-off road crash). These crashes often occur early in the morning (between 2:00 AM and 6:00 AM) in light traffic conditions.

\section{THE COPILOT (SECOND GENERATION PERCLOS MONITOR)}

The Copilot is a video-based system for measuring slow eyelid closure. The Copilot uses a structured illumination approach to identifying a driver's eyes. The approach obtains two consecutive images of the driver using a single camera. The first image (Figure 1a) is acquired using an infrared illumination source at $850 \mathrm{~nm}$ that produces a distinct glowing of the driver's pupils (the red-eye effect). The second image (Figure $1 \mathrm{~b}$ ) uses a $950 \mathrm{~nm}$ infrared illumination source that produces an image with dark pupils [Grace, 1999]. These two images are identical except for the brightness of the pupils in the image. A third image (Figure 1c) enhances the bright eyes, calculating the difference of these two images. The driver's eyes are identified in this third image by applying a threshold to the pixel brightness.

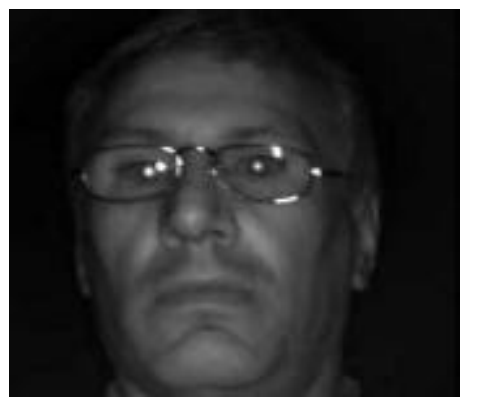

a) bright-eye image

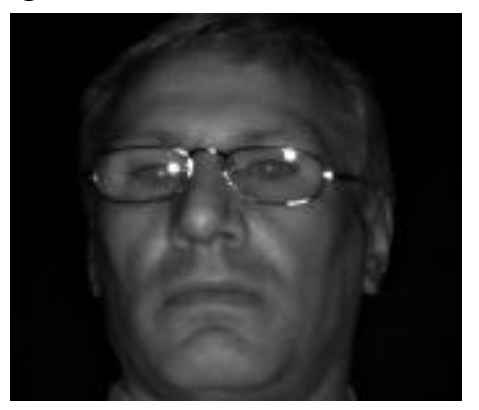

b) dark-eye image

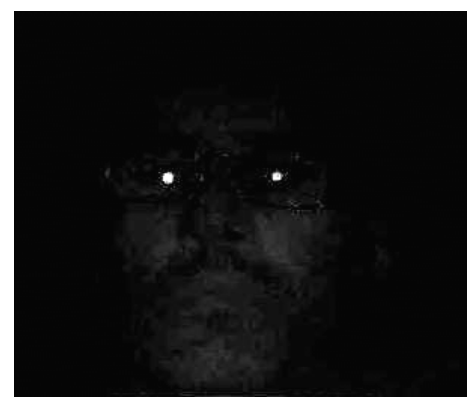

c) difference image

Figure 1: The three images used by the Copilot are shown above. The bright-eye image (a) and the dark-eye image (b) are essentially identical except for the glowing pupils in the bright eye image. The difference-image (c) eliminates all image features except for the bright pupils.

The Copilot is designed to mount on the dashboard just to the right of the steering wheel. The unit is equipped with a two degrees-of-freedom rotating base to allow the driver to adjust the system. The front face of the unit was designed to resemble the shape of a rear view mirror. The driver is instructed to adjust the aim of the unit holding it as he/she would a rear view mirror. The unit is properly adjusted when the driver can see his/her reflection in the face of the unit. The field of view is large enough to accommodate significant head movement. At a distance of $30 \mathrm{~cm}$ from the camera, a square image measuring $30 \mathrm{~cm}$ is obtained. This image size allows approximately $42 \mathrm{~cm}$ of head translation while maintaining at least one eye in the field of view. 
The integral driver interface includes a visual gauge and an audible advisory tone. The visual gauge employs six Light Emitting Diodes (LED's). The first three LED's are amber, representing moderate drowsiness levels. The second three LED's are red, representing severe drowsiness. The scale represents PERCLOS calculated over a 3-minute period where PERCLOS is defined as the proportion of time the driver's eyes are closed over a specified period. The first amber LED corresponds to $\mathrm{P} 3=0.08$. Each additional LED corresponds to an increase of 0.02 . Hence, the moderately drowsy region corresponds to $0.08 \triangleleft \mathrm{P} 3 \triangleleft .14$ and the severely drowsy range corresponds to $\mathrm{P} 3 \unrhd 14$. The audible indicator is a standard $3000 \mathrm{~Hz}$ transducer used in many automobiles. Currently, the audible advisory tone is actuated when the first amber LED is lit and again when the first red LED is lit. The audible advisory tone can also be programmed to trigger periodically when drowsiness is in the yellow or red regions.

\section{DROWSY DRIVER INTERFACE DESIGN RATIONALE}

At this time, there is insufficient scientific information to completely specify the design of the drowsy driver interface (DDI). The design of the Copilot-DDI is based on basic assumptions made regarding the nature and purpose of a DDI [Comsis, 1996; Grace, 1999; Wierwille, 1995], the results of an experiment to assess a drowsy driver interface in a driving simulation environment [Mallis, 2000] and observations of drowsiness in operational settings [Grace, 1998].

The resulting design is simple. Its provides the driver with an auditory advisory tone, reinforced by a visual gauge. These two indicators are presented in a way that is intended to encourage the driver to either take actions to increase alertness or to stop and rest.

\section{Basic Assumptions and Requirements}

- There is no substitute for sleep. The DDI should promote rest and rest breaks. It should not promote the use of the system as a "snooze alarm".

- The DDI should provide the driver with a timely notification of the progressive drowsiness onset early in the progression.

- The alarm system should provide a timely indication of sudden drowsiness onset while not providing nuisance alarms.

\section{Results of Driving Simulation Study}

A controlled experiment was undertaken with $\mathrm{n}=16$ Commercial Driving License (CDL) holders driving a high-fidelity truck simulator (TruckSim $\square$ ) to establish the effects of drowsiness feedback on: (1) driver alertness-drowsiness; (2) driving performance and (3) driver-initiated behaviors. The drowsiness feedback system tested was comprised of a PERCLOS monitor, a visual gauge, an audible advisory tone and one of two alerting stimuli. The alerting stimulus was either a voice warning alert or a peppermint scent coupled with a buzzer alert if drowsiness was sustained or reached high levels.

There is reason to believe that not all of these stimuli are required for an acceptable and effective drowsy driver interface. First, drivers' opinions regarding the accuracy and helpfulness of the interface-modes favored the auditory tone. Second, driver actions, particularly postural changes, were associated with reduced drowsiness levels. Third, drivers often did not progress from the audible indictor to the harsher alerting stimuli. This information has lead to the hypothesis that the 
audible indicator and visual gauge may be sufficient to promote self-alerting actions with a high level of acceptance by the drivers.

\section{Observations of Drowsiness}

Driver drowsiness often increases slowly over a period of hours. This is demonstrated in Figure 2 that plots PERCLOS for an over-the-road truck driver. An episode of progressive drowsiness is observed in Figure $2 \mathrm{a}$ from 0:45 to 1:45. Sudden drowsiness onset is also observed. Figure $2 \mathrm{~b}$ shows a driver who in two minutes progressed from PERCLOS $=0.01$ to PERCLOS $=0.8$ at approximately 23:00.

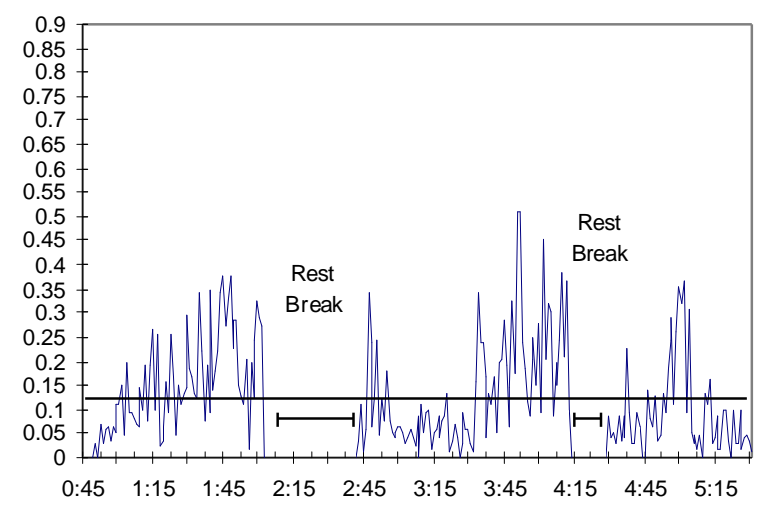

(a) chronic - progressive drowsiness

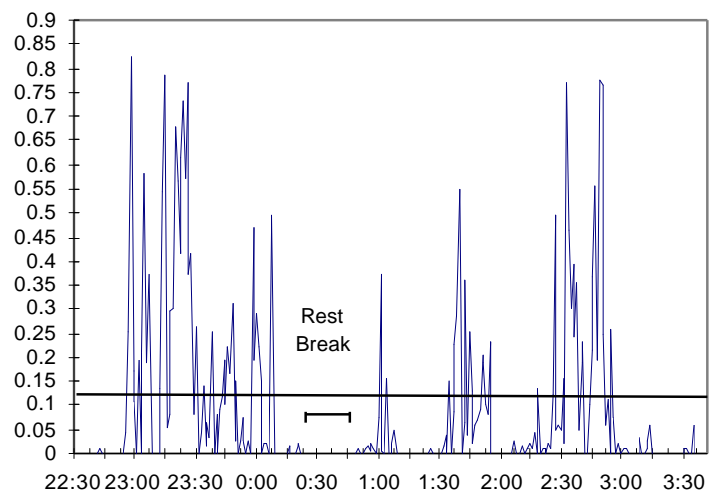

(b) acute - sudden drowsiness

Figure 2: Progressive drowsiness onset and sudden drowsiness onset are shown above.

\section{OPERATIONAL ISSUES}

It is vital that a drowsiness monitor be used within a management structure that promotes an understanding of fatigue, the potential consequences of driving while drowsy and provides guidance to drivers for managing fatigue. The common thread for addressing fatigue in any work environment is basic human physiology, in particular the need for sleep and the influence of natural circadian rhythms. If drivers are obtaining sufficient sleep, their alertness and performance will be optimized. If, however, drivers are receiving insufficient sleep, their alertness and performance will degrade accordingly. A driver's alertness and performance levels will also cycle with the normal circadian rhythms. Performance degradation is most likely during the natural circadian low points that occur for most of us between 2:00 AM and 6:00 AM.

A number of management structures have been proposed to promote fatigue management, safety and responsibility on the part of the driver [Dinges, 1998; Grace, 1999]. However, fatigue is illusive and often ill-defined, making management of fatigue qualitative and often based on indirect measures such as total time off-duty, etc. The problem is that decision-makers are often faced with too few facts and too many options [Barfoot, 1998]. The Copilot can provide the driver and management with objective quantitative measures of driver drowsiness providing a means for assessing the effectiveness of a fatigue management program.

The feedback from the Copilot can also assist a driver in determining the best time to stop and rest. If, for example, the driver receives historical data that shows he/she is often drowsy between 3:00 AM and 4:00 AM, he/she may decide to adjust his/her schedule to avoid driving at that time. Furthermore, the immediate feedback provided by the Copilot can encourage drivers to stop and rest using one of the napping strategies taught as part of a fatigue management training course [Oneil, 1996]. The Copilot can be used in certain circumstances to extend a drive until the driver reaches a 
safe place to stop. Since a driver may need to travel for 20 minutes or longer to find a safe place, the Copilot can potentially help a driver maintain alertness during this critical period.

\section{CONCLUSIONS}

The Copilot is a low-cost drowsiness monitor intended for use in commercial operations involving nighttime driving. The system is the outcome of over ten years of research sponsored by the National Highway Traffic Safety Administration and the Federal Motor Carrier Safety Administration. The unit is designed for robust operation in a heavy truck environment. Work is continuing to validate the Copilot, to refine the driver interface and to determine the best practices for using the monitor.

\section{ACKNOWLEDGEMENTS}

This work has been funded by the National Highway Traffic Safety Administration and the Federal Motor Carrier Administration

\section{REFERENCES}

Barfoot, G., Quantitative Methods for Organizational Decision-Making, http://www.tgsa.edu/online/cybrary/gbarfoot.html, August 1998

Comsis Corporation: Preliminary Human Factors Guidelines for Crash Avoidance Warning Devices, NHTSA Project No. DTNH22-91-C-07004, 1996

Dinges, D. F., Mallis, M. M., Maislin, G., Powell, J.W. Evaluation of Techniques for Ocular Measurement as an Index of Fatigue and as the Basis for Alertness Management, DOT HS 808 762, April, 1998.

Dinges, D. F., Mallis, M. M., “Managing fatigue by drowsiness detection: Can technological promises be realized?" in Hartley, Laurence (Ed.) “Managing Fatigue in Transportation” pg 209229, 1998

Grace, R., Benjamin, A. L., Application of a Heavy Vehicle Drowsy Driver Detection System, Proceedings of the SAE International Truck \& Bus Meeting and Exposition, November 15-17, 1999

Grace, R., Byrne, V. E., Legrand, J. M., Gricourt, D. J., Davis, R. K., Staszewski, J. J., Carnahan, B., A Machine Vision Based Drowsy Driver Detection System for Heavy Vehicles, Proceedings of The Ocular Measures of Driver Alertness Conference, pp 75-86, April 26-27, 1999, FHWA-MC-99-136

Grace, R., Byrne, V.E., Legrand, J.M., Gricourt, D.J., Davis, R.K., Staszewski, J.J., Carnahan, B., A Drowsy Driver Detection System for Heavy Vehicles, Proceedings of the Digital Avionics Systems Conference (DASC), Belleview, Washington, Oct. 31-Nov. 5, 1998.

Mallis, M. M., Maislin, G., Konowal, N., Bierman, D. M., Byrne, V. E., Davis, R. K., Grace, R., Dinges, D. F., Biobehavioral Responses to Drowsy Driving Alarms and Alerting Stimuli, Final Report DOT Contract No. DTNH22-93-D-07007, March 2000

Oneil, T. R., Krueger, G. P., Van Hemel, S. B., Understanding Fatigue and Alert Driving, American Trucking Associations, October, 1996

Wierwille, WW, Fayhey, S. E., Fairbanks, RJ, Kirn, CL: Research on Vehicle-Based Driver Status/Performance Monitoring: Development: Seventh Semiannual Report. National Highway Traffic Safety Administration Final Report: DOT HS 808 299, 1995 